\title{
The Fourth International Workshop on the Standardisation of Insulin Autoantibody Measurement
}

\author{
B. Kuglin, H. Kolb, C. Greenbaum, N. K. Maclaren, Å. Lernmark, and J. P. Palmer \\ on Behalf of the Immunology and Diabetes Workshops and Participating Laboratories**
}

During the course of previous insulin autoantibody (IAA) workshops [1-3] it had become apparent that fluid phase (RIA) and solid phase (ELISA) antibody detection assays gave discordant results for many sera. It was therefore decided to compare the disease association of IAA as determined by RIA vs ELISA in the Fourth IAA Workshop.

For this purpose 31 participating laboratories submitted unselected sera obtained from healthy control subjects $(n=61)$, from patients with newly diagnosed Type 1 (insulin-dependent) diabetes mellitus $(n=30)$ prior to or within $24 \mathrm{~h}$ after the first insulin injection, and from relatives of Type 1 diabetic patients $(n=30)$. The sera were aliquoted and distributed by the Immunology of Diabetes Workshops (IDW) Laboratory in Gainesville, USA. Results from 18 fluid phase and from 10 solid phase assays could be included in the analysis. In both assays sera were tested twice, before and after competitive inhibition with excess insulin. Absolute counts of immunoglobulin bound iodinated insulin or absolute optical densities and/or displaceable "specific" signals (delta \% binding or delta optical density) were submitted. The distribution of displaceable signals obtained with the 61 normal sera is shown in Figure 1 for two representative laboratories and no relevant differences were observed between the fluid phase and solid phase assays. RIA and ELISA gave similarly rare positive readings with control sera. Most of the RIA laboratories $(16$ of $18 ; 89 \%$ ) read none or only one control sera as being above 3 SD for all controls and 7 of $10(70 \%)$ laboratories determined 0 or 1 control sera as above 3 SD by ELISA. Signals higher than 5 SD were detected in none of the control sera by RIA and in one serum by $2(20 \%)$ out of 10 ELISAs.

* A.Arnaiz-Villena (Madrid, Spain), C.E.de Beaufort (Luxembourg), D.Becker (Pittsburgh, USA), F.Becker (Giessen, FRG), C. Betterle (Padua, Italy), E. Bosi (Milan, Italy), P.Colman (Melbourne, Australia), E. B. Dean (London, UK), N. Dozio (Milan, Italy), G.S. Eisenbarth (Boston, USA), R. B. Elliott (Auckland, New Zealand), E.A.M.Gale (London, UK), F. Gorus (Brussels, Belgium), A. Hübinger (Düsseldorf, FRG), R. Humbel (Luxembourg), J.Karjalainen (Oulu, Finland), H.Keilacker (Karlsburg, GDR), H. Kolb (Düsseldorf, FRG), B. Kuglin (Düsseldorf, FRG), J. Kwan (Toronto, Canada), C.Levy-Marchal (Paris, France), N. Maclaren (Gainesville, USA), J.M. Martín-Villa (Madrid, Spain), J.P.Palmer (Seattle, USA), K.M.Reinauer (Tübingen, FRG), W. Riley (Gainesville, USA), W. Scherbaum (Ulm, FRG), G.J.P.Singh (Newport Beach, Canada), J.C.Sodoyez (Liege, Belgium), C.H.Thivolet (Lyon, France), B.Vialettes (Marseille, France), M. A.Wai-Sai (Newport Beach, Canada), T.J. Wilkin (Southampton, UK), R. Zanchetta (Padua, Italy), A.-G.Ziegler (Munich, FRG)
In sera from newly onset Type 1 diabetic patients the RIA results showed significantly higher specific signals than those obtained by ELISA. A representative positive serum (positive in 26 of 28 assays) yielded a mean of $178 \mathrm{SD}$ (range 12.0-536) by RIA vs 5.8 SD (range 1.3-16.7) by ELISA. This in part reflects higher background and a corresponding variation of signals for control sera in ELISA, i.e. both the mean signal in normal sera and the mean variance, are higher in ELISA assays (Fig. 2). Because of the higher specific signal and the concomitantly greater number of positive results in patient sera it was conchuded that fluid phase assays were superior to solid phase assays for identifying disease associated IAA signals.

There was little difference in the height of the specific signal obtained by the various RIAs for a given serum, classification of sera as positive or negative largely dependend on the relation of the signal to the 3 SD cut off, i.e., RIAs with small variation of signals in control sera had a small mean SD. This led to a lower cut off and higher SD scores for IAA positive sera. Most of the radiobinding assay characteristics were identical or closely related to recently published methods [4-6] and are summarized in Table 1 . There was agreement that IAA as measured by RIA should be included in the Proficiency Program sponsored by the Immunology of Diabetes Workshops and that IAA results might be made comparable between laboratories.

The 5th International Workshop on the Standardization of IAA Measurement will test the practicability and reliability of the introduction of IAA units defined by a standard serum. To participate in this hopefully final stage of IAA standardization please contact the following Workshop organizers:

Dr. J.Palmer, Seattle VA Medical Center, Endocrinology, $1660 \mathrm{~S}$ Columbian Way, Seattle, WA 98108, USA

Dr. T. J. Wilkin, Dept. Medicine II, Level D South Block, Southampton General Hospital, Southampton S09 4XY, UK

To participate in the Proficiency Test please contact:

Dr. N. K. Maclaren, Department of Pathology, Box J-275, College of Medicine, University of Florida, Gainesville, FL 32610-0275, USA.

Acknowledgements. We thank members of the participating laboratories, in particular Drs. L. Castano and J. C. Sodoyez for the discussion of results.

Table 1. Radioimmunoassay - assay characteristics

\begin{tabular}{ll}
\hline Total volume & $0.15-0.50 \mathrm{mI}$ \\
Final serum concentration & $1: 1-1: 10$ \\
Incubation time & $18 \mathrm{~h}-168 \mathrm{~h}$ \\
Specific activity of ${ }^{125}$ I insulin & $75-374 \mathrm{mCl} / \mathrm{mg}$ \\
c.p. m./tube & $5000-20000$ \\
Amount of cold insulin for displacement & $0.5 \mathrm{mg} / 1-120 \mathrm{mg} / 1$ \\
\hline
\end{tabular}




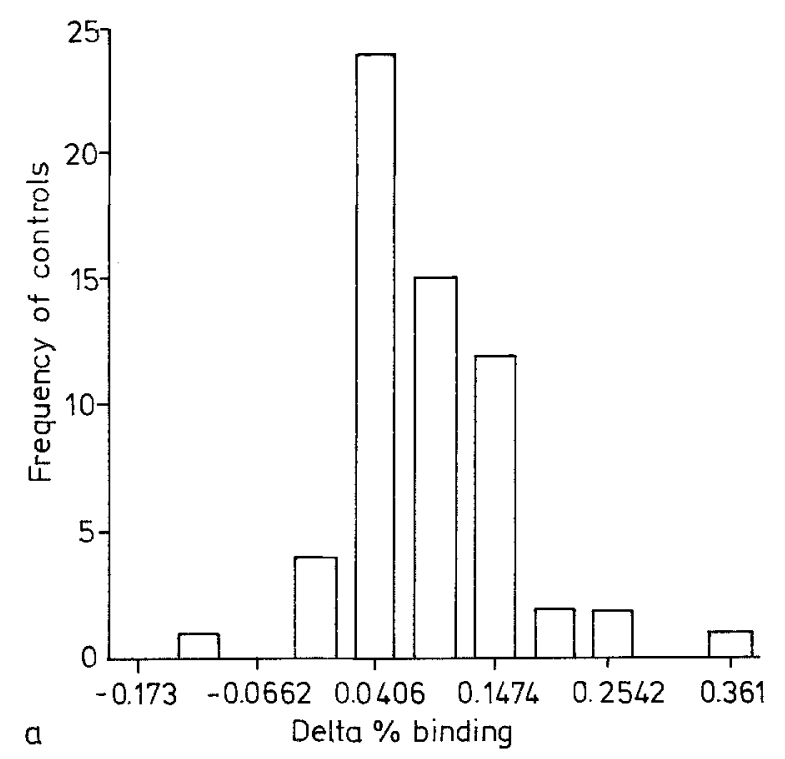

1 $\square$ RIA

$2 a$

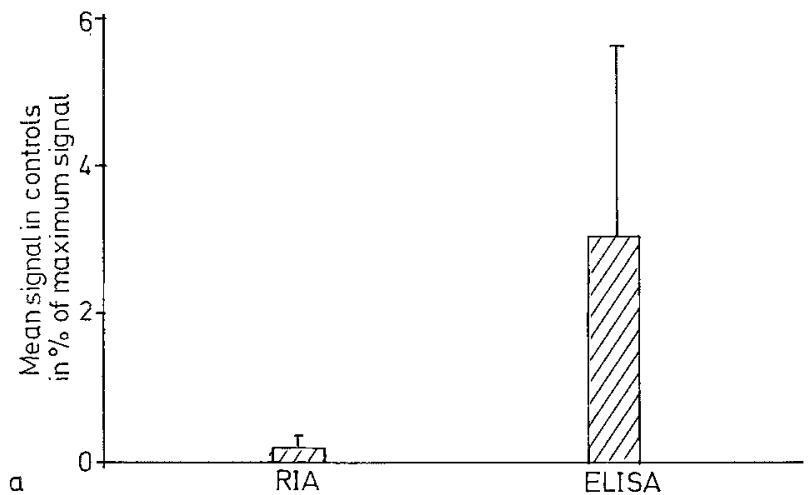

Fig.1a, b. The distribution of the specific signal in sera from 61 healthy control subjects in two representative laboratories. a Distribution of control sera in a representative ELISA laboratory. Results are given as delta optical density. b Distribution of control sera in a representative RIA laboratory. Results are given as delta percent binding

Fig. 2 a, b. In sera from 61 control subjects both, the mean background signal (a) and the mean variance (b) are strongly higher in ELISAs than in RIAS. Results are given as percentage of maximal assay signal

\section{References}

1. Wilkin TJ, Palmer J, Bonifacio E, Diaz J-L, Kruse V (1987) First international workshop on the standardisation of insulin autoantibodies. Diabetologia 30: 676-677

2. Wilkin TJ, Palmer J, Kurtz AB, Bonifacio E, Diaz J-L (1988) The second international workshop on the standardization of insulin autoantibody (IAA) measurement. Diabetologia 31: 449-450
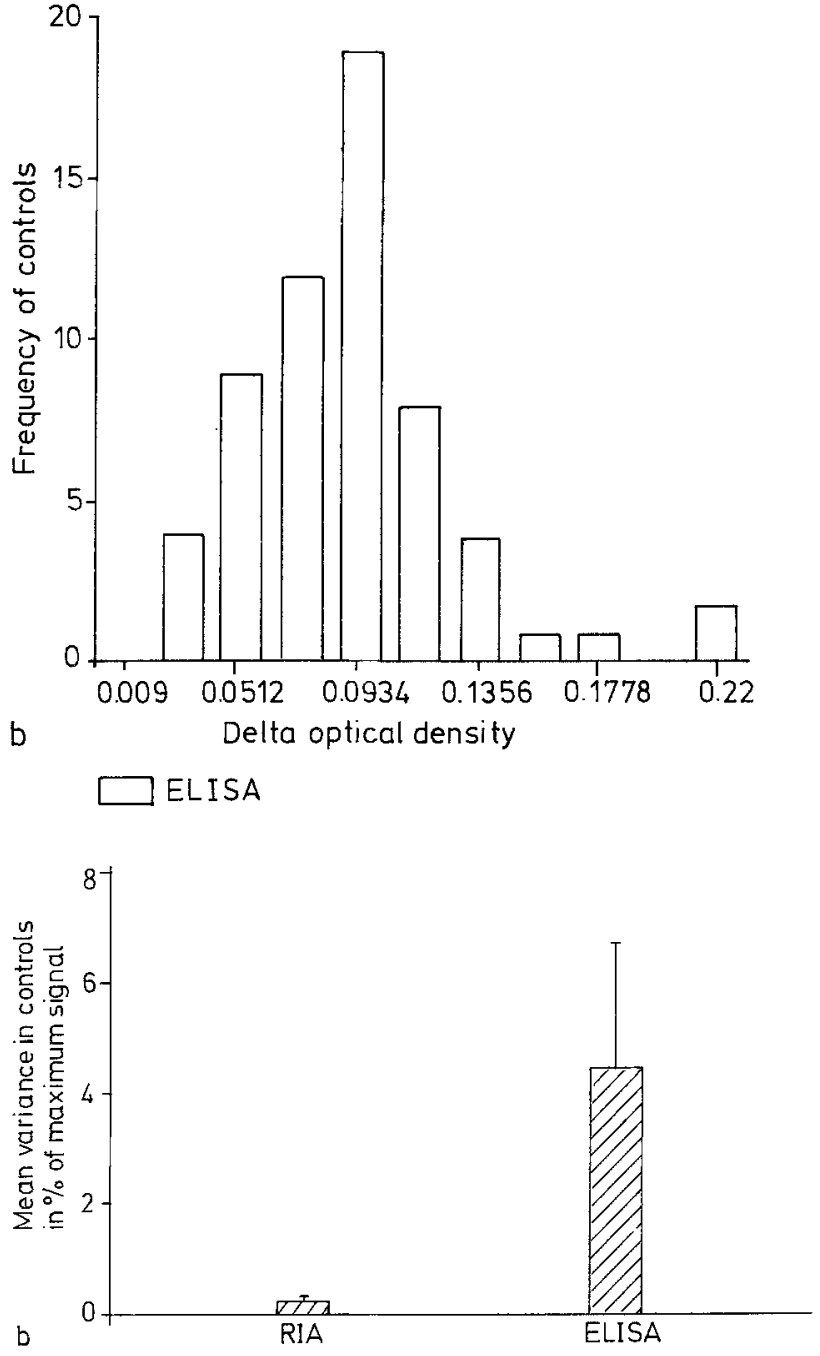

3. Palmer JP, Wilkin TJ, Kurtz AB, Bonifacio E (1990) The third international workshop on the standardization of insulin autoantibody measurement. Diabetologia 33: 60-61

4. Vardi P, Dib SA, Tuttleman M, Connelly JE, Grinbergs M, Radizabeh A, Riley WJ, Maclaren NK, Eisenbarth GS, Soeldner JS (1987) Competitive insulin autoantibody assay. Prospective evaluation of subjects at high risk for development of type 1 diabetes mellitus. Diabetes 36: 1286-1291

5. Palmer JP (1987) Insulin autoantibodies: their role in the pathogenesis of IDDM. Diabetes/Metabol Rev 3:1005-1015

6. Atkinson MA, Maclaren NK, Riley WJ, Winter WE, Fisk DD, Spillar RP (1986) Are insulin autoantibodies markers for insulin dependent diabetes mellitus? Diabetes 35: 894-897

Prof. Dr. H. Kolb

Diabetes Research Institute

University of Düsseldorf

Auf'm Hennekamp 65

D-4000 Düsseldorf 1

FRG 